\title{
Concept of Complex-Valued Parametric Pulse Models
}

\author{
Klaus Pourvoyeur, ${ }^{1}$ Andreas Stelzer, ${ }^{2}$ and Gerald Oßberger ${ }^{1}$ \\ ${ }^{1}$ Linz Center of Mechatronics GmbH, Altenberger Straße 69, 4040 Linz, Austria \\ ${ }^{2}$ Institute for Communications and Information Engineering, Johannes Kepler University, Altenberger Straße 69, 4040 Linz, Austria
}

Received 4 February 2005; Revised 7 November 2005; Accepted 8 November 2005

Recommended for Publication by Geert Leus

In pulse-based radar systems, the knowledge of certain parameters of the received radar pulse is of great importance. We introduce a complex-valued parametric pulse model by extending a real-valued pulse signal into the complex plane. A modulation angle parameter unique to the complex representation gives an additional degree of freedom and can be used to model the basic shape of the pulse, thus lifting the conventional restriction to fixed pulse shapes in real-valued correlation techniques. As physical signals are real valued, the imaginary part of the complex signal is calculated by using the Hilbert transformation. Parameter estimation is based on the complex-valued continuous wavelet transform. The main advantages of this concept are demonstrated on synthetic data and verified on ultrawideband pulse radar measurements.

Copyright (C) 2006 Klaus Pourvoyeur et al. This is an open access article distributed under the Creative Commons Attribution License, which permits unrestricted use, distribution, and reproduction in any medium, provided the original work is properly cited.

\section{INTRODUCTION}

For the detection and characterization of a pulse covered in noise, conventional correlation methods like matched filtering with real-valued signal shapes suffer one major drawback in analyzing the sampled signal: the requirement of the exact knowledge of the pulse shape. Since this information is generally not available in pulse-based radar systems, the employed signal analysis method must provide enough flexibility to deal with this uncertainty. The wavelet transform (WT) that is based on correlation with translated and dilated versions of a basic template waveform, or wavelet, is a means of overcoming some of the restrictions of the matched filtering approach, yet the analysis wavelet has to be chosen properly to match the specific shape of the searched pulse.

In this contribution, we discuss the concept of complexvalued parametric pulse (CVPP) models in combination with the WT that gives us the ability to deal with different pulse shapes with a priori knowledge of only the approximate characteristic of the pulse. Since signals in nature are always real valued, the imaginary part of a CVPP has to be estimated from its real part.

This paper is organized as follows. Section 2 describes the basic idea of complex-valued pulse models. Section 3 discusses the basic ideas of CVPPs, Section 4 explains how parameter estimation is accomplished, Section 5 deals with the main advantages of CVPPs on synthetic signals, and
Section 6 verifies the potential of CVPPs on measured data. A summary of the paper is given in Section 7 .

\section{COMPLEX EXTENSION}

The concept of CVPPs uses the Hilbert transform to estimate the imaginary part of the pulse based on its real part. The Hilbert transform, defined by

$$
H\left\{f_{h}(t)\right\}=\frac{1}{\pi} \int_{-\infty}^{\infty} \frac{f_{h}(t)}{t-\tau} d \tau,
$$

is a linear transform and rotates the phase of all spectral components of the original signal by $\pi / 2 \mathrm{rad}$. In time domain, the real-valued signals, $f_{h}(t)$ and $H\left\{f_{h}(t)\right\}$, are orthogonal. The complex-valued signal $f_{h}^{+}(t)$ whose imaginary part is the Hilbert transform of its real part $f_{h}(t)$,

$$
f_{h}^{+}(t)=f_{h}(t)+j H\left\{f_{h}(t)\right\},
$$

is called the analytic signal. The Fourier spectrum of the analytic signal is double that of the original signal at positive frequencies and zero at negative frequencies. Furthermore, the energies of the original signal and its Hilbert transform are identical. The energy conservation of the Hilbert transform is especially of importance in regard to scaling and pulse modeling. For additional information concerning the Hilbert transform, we refer to [1]. 


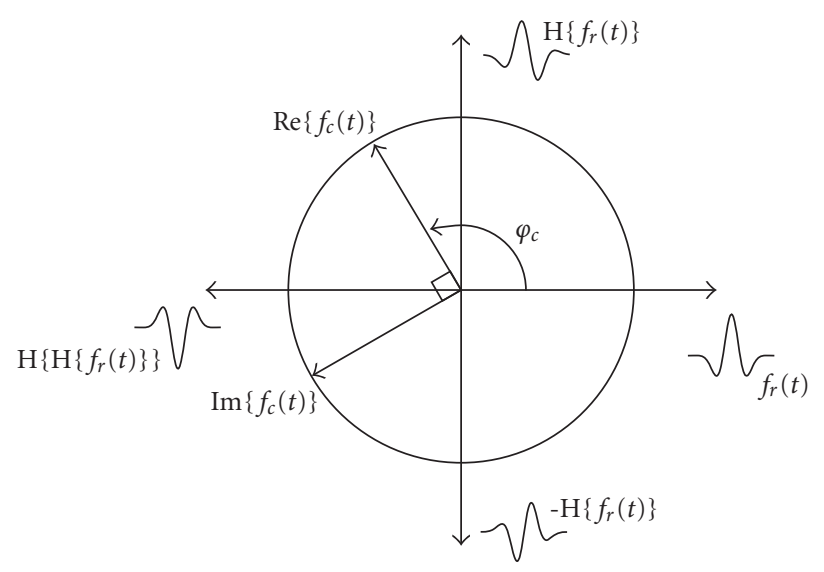

FIGURE 1: Geometrical interpretation for the pulse-shape forming of the modulation angle $\varphi_{c}$ and the Hilbert transform $H\{\cdot\}$ for the real and imaginary parts of a complex-valued pulse $f_{c}(t)$.

We define a modulated complex pulse $f_{c}(t)$, consisting of a real part $f_{r}(t)$ and an imaginary part $f_{i}(t)$ related to one another by the Hilbert transform as well as a modulation term $e^{-j \varphi_{c}}$ by

$$
f_{c}(t)=\left(f_{r}(t)+j f_{i}(t)\right) e^{-j \varphi_{c}}=f_{r}^{+}(t) e^{-j \varphi_{c}} .
$$

The real and imaginary parts of the complex pulse $f_{c}(t)$ of (3) are given by

$$
\begin{aligned}
\operatorname{Re}\left\{f_{c}(t)\right\} & =f_{r}(t) \cos \left(\varphi_{c}\right)+H\left\{f_{r}(t)\right\} \sin \left(\varphi_{c}\right), \\
\operatorname{Im}\left\{f_{c}(t)\right\} & =H\left\{f_{r}(t)\right\} \cos \left(\varphi_{c}\right)-f_{r}(t) \sin \left(\varphi_{c}\right) .
\end{aligned}
$$

The complex-valued modulation term $\mathrm{e}^{-j \varphi_{c}}$ rotates the complex-valued pulse $f_{c}(t)$ in the complex plane by the modulation angle $\varphi_{c}$, which can be interpreted as the phasing of the pulse. Transforming the real part $\operatorname{Re}\left\{f_{c}(t)\right\}$ of the complex pulse $f_{c}(t)$ leads to

$$
\begin{aligned}
H\left\{\operatorname{Re}\left\{f_{c}(t)\right\}\right\} & =H\left\{f_{r}(t)\right\} \cos \left(\varphi_{c}\right)+H\left\{H\left\{f_{r}(t)\right\}\right\} \sin \left(\varphi_{c}\right) \\
& =\operatorname{Im}\left\{f_{c}(t)\right\} .
\end{aligned}
$$

Therefore, the Hilbert transform is suitable for the estimation of the imaginary part of the complex pulse $\operatorname{Im}\left\{f_{c}(t)\right\}$ by transforming its real part $\operatorname{Re}\left\{f_{c}(t)\right\}$ without making any systematical error even for an unknown modulation angle $\varphi_{c}$. Since measured time-domain pulses are always real valued, this property is of enormous importance. A geometrical interpretation for the pulse-shape forming of (3) for an arbitrary pulse is shown in Figure 1.

\section{PARAMETRIC PULSE MODEL}

Beside the complex extension, the basic idea of a CVPP relies on the WT. The WT represents a time function on the timescale plane. The modern concept of wavelets was founded in the early 90's by Grossman and Morlet in the field of analyzing seismic data [2]. The WT of a possibly complexvalued function $f_{w}(t)$ to the possibly complex-valued mother wavelet $\psi(t)$ is defined by

$$
W_{f_{w}}(\tau, a)=\int_{-\infty}^{\infty} f_{w}(t) \frac{1}{\sqrt{a}} \psi^{*}\left(\frac{t-\tau}{a}\right) d t
$$

with $a$ as the dilatation factor and $\tau$ as the translation factor. Based on the normalized mother wavelet $\psi(t)$, the WT correlates the signal $f_{w}(t)$ with a scaled, shifted, and stretched version of this wavelet $\psi(t)$. The dilatation factor $a$ of a wavelet defines its active width and is therefore a quantum for its resolution. The scaling factor $a^{-1 / 2}$ normalizes the energy of the wavelet,

$$
\|\psi(t)\|_{2}=\frac{1}{\sqrt{a}}\left\|\psi\left(\frac{t}{a}\right)\right\|_{2}
$$

The Euclidian norm is defined by

$$
\|\psi(t)\|_{2}=\sqrt{\int_{-\infty}^{\infty} \psi(t) \psi^{*}(t) d t},
$$

with $\psi^{*}(t)$ denoting the conjugate complex of $\psi(t)$. The translation factor $\tau$ indicates the point in time at which the wavelet is localized. Furthermore, the WT is linear as well as time invariant, and the energy contained in the signal is preserved. For further information concerning the WT, we refer to $[3,4]$.

The CVPP $f_{c}(t)$ we propose is based on a scaled, shifted, stretched, and modulated version of the complex-valued analytic mother wavelet $\psi_{c}(t)=\psi_{r}^{+}(t)$,

$$
f_{c}(t)=f_{r}^{+}(t) e^{-j \varphi_{c}}=\frac{1}{\sqrt{a_{c}}} k_{c} \psi_{r}^{+}\left(\frac{t-\tau_{c}}{a_{c}}\right) e^{-j \varphi_{c}},
$$

and is described by four parameters: (1) the intensity coefficient $k_{c}$, (2) translation $\tau_{c}$, (3) dilatation $a_{c}$ of the mother wavelet, and (4) the modulation angle $\varphi_{c}$. The mother wavelet itself can be both parametric or nonparametric. Without the modulation angle parameter $\varphi_{c}$, the basic shape of the mother wavelet would determine the basic shape of the radar pulse to be searched. A typical nonparametric wavelet is a measured and smoothed reference signal. Very common parametric wavelets to model, for example, ultrawideband (UWB) radar pulses, directly are the Gauss wavelets $\psi_{g}(t)$ defined by the $p$ th derivative of the normalized Gaussian bellshape curve [5] that is given by

$$
\psi_{g}(t)=C_{g}\left(e^{-t^{2}}\right)^{(p)},
$$

where the factor $C_{g}$ normalizes the energy of these wavelets. Gauss wavelets $\psi_{g}(t)$ are also a proper choice for the real part of the complex-valued analytic mother wavelet $\psi_{r}^{+}(t)$ forming a CVPP. The first two representatives of this wavelet class for $p=1$ and $p=2$ are called Gaussian monocycle and Ricker wavelet.

The real part $\operatorname{Re}\left\{f_{c}(t)\right\}$ of a CVPP formed by the Ricker wavelet for $k_{c}=1, \tau_{c}=0$, and $a_{c}=1$ is shown in Figure 2 for $\varphi_{c}=0^{\circ}, 30^{\circ}, 60^{\circ}$, and $90^{\circ}$. Obviously for $\varphi_{c}=0^{\circ}$, the real part $\operatorname{Re}\left\{f_{c}(t)\right\}$ of the CVPP is identical to the Ricker wavelet, and for $\varphi_{c}=90^{\circ}$, it is identical to the Hilbert transform of the Ricker wavelet. 


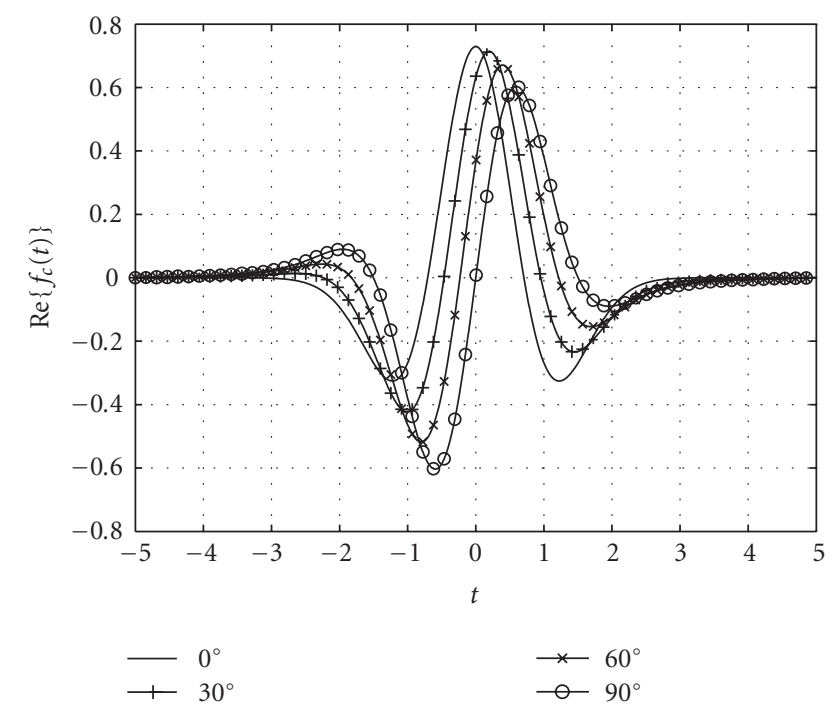

FIgURe 2: Real part $\operatorname{Re}\left\{f_{c}(t)\right\}$ of a CVPP formed by the Ricker wavelet for $k_{c}=1, \tau_{c}=0, a_{c}=1$, and $\varphi_{c}=0^{\circ}, 30^{\circ}, 60^{\circ}$, and $90^{\circ}$.

\section{PARAMETER ESTIMATION}

When using a wavelet of Gauss type for pulse analysis, the wavelet order should comply with the number of extrema of the searched pulse minus one. In the real-valued case, the order of the Gauss wavelet has to be chosen very carefully because it is the only parameter to model the basic shape of the wavelet. Unfortunately, the Gauss-type wavelets allow only the modeling of even or odd functions, hybrid forms are not included in this class. Furthermore, if the basic order (even or odd) of the pulse is mismatched, the results degrade dramatically as will be discussed in Section 5 .

In the complex-valued case, the choice of the order is relatively uncritical. In this case, the order of the wavelet is mostly important for modeling the pre- and postoscillations of the pulse. Owing to the modulation angle $\varphi_{c}$, the CVPPs also include hybrid forms even though the real part of the basic wavelet for pulse modeling is only an even or an odd function.

The measured signal can be interpreted as the real part $\operatorname{Re}\left\{f_{c}(t)\right\}$ of the analytical function $f_{c}(t)$. The best correlation between the scaled, shifted, and stretched wavelet $\psi_{c}(t)$ and the complex-valued signal $f_{c}(t)$ is achieved if the wavelet matches with the pulse in the signal. Hence, the translation factor $\tau_{c}$ and the dilatation factor $a_{c}$ can be computed by

$$
\left(\tau_{c}, a_{c}\right)=\arg \max _{(\tau, a)}\left|W_{f_{c}}(\tau, a)\right| .
$$

The optimization problem of (11) can be solved by a numerical gradient method. Unfortunately, for real-valued pulses, as will be shown in Section 6, the absolute value of the wavelet transform even for one single pulse shows several undesired side maxima. Therefore, several starting points have to be chosen. The calculation complexity of the complex-valued wavelet transform is slightly increased compared to the real-valued case. Advantageously, a single complex-valued pulse shows no undesired side maxima, whereby only one starting point for a gradient method is sufficient. Therefore, the overall calculation time in the complex case is even reduced.

For UWB pulse applications, as discussed in [6-8], the very large bandwidth of the pulse results in an extremely short duration of the pulses in time domain. The sampling of such short pulses requires high-speed sampling oscilloscopes; from a signal processing point of view, the absolute time base is of no significance.

If the dilatation factor of the mother wavelet is known, the WT has to be performed only for one single scale. For a complex-valued wavelet and/or a complex-valued signal, the limitation of the WT to a single scale corresponds to a complex-valued matched filter.

The WT at time $\tau_{c}$ and at scale $a_{c}$ is calculated to

$$
\begin{aligned}
W_{f_{r}^{+}}\left(\tau_{c}, a_{c}\right) & =\int_{-\infty}^{\infty} \operatorname{Re}\left\{f_{c}(t)\right\}^{+} \frac{1}{\sqrt{a_{c}}} \psi_{c}^{*}\left(\frac{t-\tau_{c}}{a_{c}}\right) d t \\
& =\int_{-\infty}^{\infty} f_{c}(t) \frac{1}{\sqrt{a_{c}}} \psi_{c}^{*}\left(\frac{t-\tau_{c}}{a_{c}}\right) d t \\
& =\int_{-\infty}^{\infty} \frac{1}{\sqrt{a_{c}}} k_{c} \psi_{c}\left(\frac{t-\tau_{c}}{a_{c}}\right) e^{-j \varphi_{c}} \frac{1}{\sqrt{a_{c}}} \psi_{c}^{*}\left(\frac{t-\tau_{c}}{a_{c}}\right) d t \\
& =k_{c} e^{-j \varphi_{c}} \|\left.\frac{1}{\sqrt{a_{c}}} \psi_{c}\left(\frac{t-\tau_{c}}{a_{c}}\right)\right|_{2} ^{2} \\
& =k_{c} e^{-j \varphi_{c}} .
\end{aligned}
$$

As we can see, the WT for scale $a_{c}$ and translation factor $\tau_{c}$ indicates directly the intensity factor $k_{c}$ as well as the modulation angle $\varphi_{c}$ of the complex pulse model. Hence, a priori knowledge of the modulation angle $\varphi_{c}$ is unnecessary for a perfect correlation in time domain.

Like in any correlation-based method, the parameter estimation for the CVPPs is very immune to white Gaussian noise covering the signal. Compared to real-valued correlation methods, the impact of noise on the CVPP is very similar. There is no additional information and therefore also no reduction in the noise behavior, because the imaginary part is calculated from the real-valued signal and not measured separately.

\section{SIMULATIONS}

The basic properties of a complex-valued correlation on a CVPP compared to a real-valued correlation on a real-valued function are demonstrated on synthetic data without noise. For a better clarity, it is assumed that the dilatation factor of the pulse is known, hence the WT has to be computed only on a single scale. As discussed above, restraining the WT to a single scale is similar to the matched filter concept.

The absolute value of the normalized cross-correlation function (CCF) between the real-valued Ricker wavelet and the real part $\operatorname{Re}\left\{f_{c}(t)\right\}$ of a CVPP formed by the Ricker wavelet for $k_{c}=1, \tau_{c}=0, a_{c}=1$, and $\varphi_{c}=0^{\circ}$ as well as $90^{\circ}$ is shown in Figure 3. For $\varphi_{c}=0^{\circ}$, the maximum of the absolute value of the normalized CCF is achieved at the 


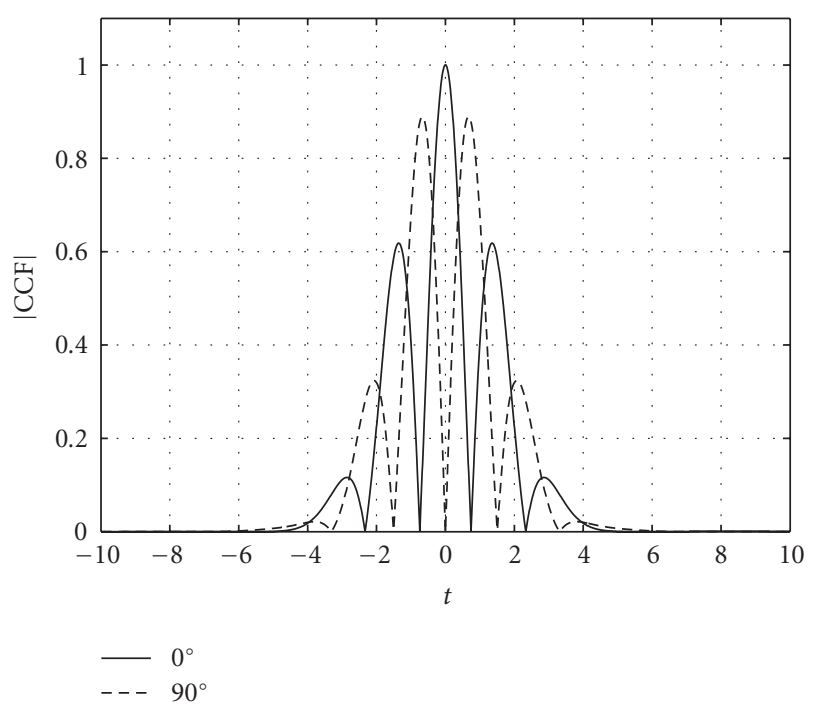

FIGURE 3: Absolute value of the normalized CCF between the Ricker wavelet and the real part $\operatorname{Re}\left\{f_{c}(t)\right\}$ of a CVPP formed by the Ricker wavelet for $k_{c}=1, \tau_{c}=0, a_{c}=1$, and $\varphi_{c}=0^{\circ}$ as well as $90^{\circ}$.

pulse position. As $\varphi_{c}$ diverges from the value of $0^{\circ}$, the peak maximum decreases in amplitude and deviates from the true position, until for $\varphi_{c}=90^{\circ}$, the CCF at the pulse position is equal to zero; while simultaneously two relatively large side maxima appear to the left and to the right of the correct location, thus inhibiting unambiguous position estimation. This behavior is explained by the fact that the CCF is the correlation of two even functions for $\varphi_{c}=0^{\circ}$, and that of an odd and an even functions for $\varphi_{c}=90^{\circ}$. In any case, the absolute value of the normalized CCF shows several side maxima that are not located at the pulse position. This example illustrates the need of the knowledge of the exact pulse shape for a real-valued correlation.

The absolute value of the normalized CCF between the analytic Ricker wavelet and the analytical signal $f_{c}(t)$ of a CVPP formed by the Ricker wavelet for $k_{c}=1, \tau_{c}=0, a_{c}=1$, and $\varphi_{c}=0^{\circ}$ as well as $90^{\circ}$ is shown in Figure 4 The modulation angle has completely no influence on the absolute value of the CCF. Furthermore, the plot shows no undesired side maxima beside the main peak because the complex correlation between an analytic signal and an analytic wavelet is still analytic. Hence, the absolute value of this CCF can be interpreted as the envelope of the pulse.

\section{MEASUREMENTS}

The precise pulse analysis for a wide field of applications is still a challenge. The basic use of a complex-valued correlation method for signal analysis is, for example, discussed in [9]. Another field of pulse-based radar applications is the detection of low metal content antipersonnel mines buried in lossy ground. The dispersion behavior of natural ground material results in a change of duration and shape of the transmission pulse. In [10], a pulse reconstruction method

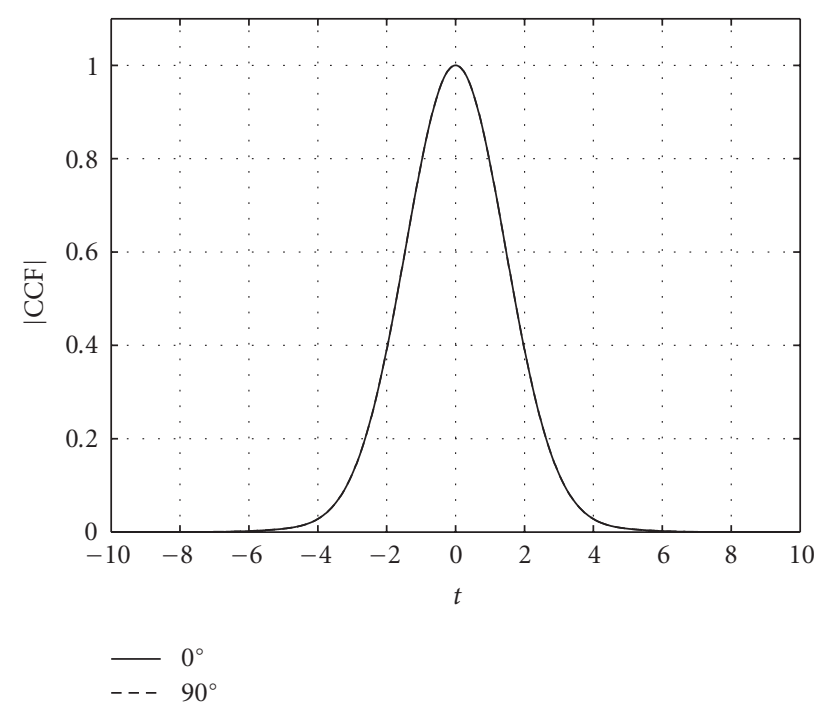

FIgUre 4: Absolute value of the normalized CCF between the analytic Ricker wavelet and the analytical real part $\operatorname{Re}\left\{f_{c}(t)\right\}^{+}$of a CVPP formed by the Ricker wavelet for $k_{c}=1, \tau_{c}=0, a_{c}=1$, and $\varphi_{c}=0^{\circ}$ as well as $90^{\circ}$.

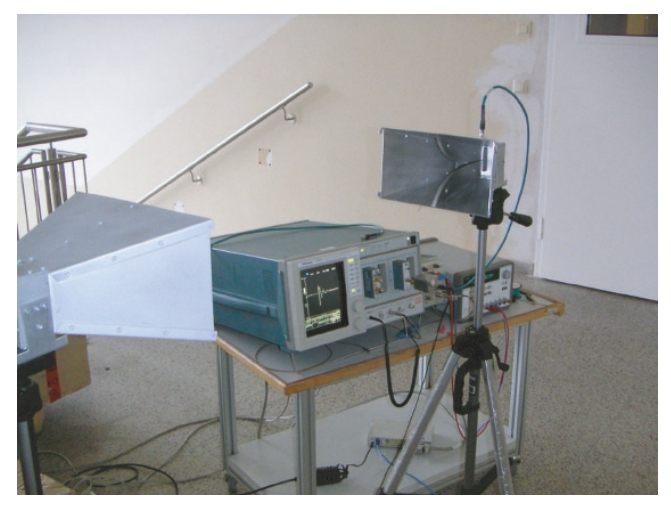

FIgURE 5: Photo of the UWB pulse radar measurement test setup.

based on the real-valued WT is used for this purpose. The application of a CVPP is demonstrated on UWB pulse measurements. For this purpose, a pulse radar test setup was built to generate real radar reflection data. The test setup consists of a pulse generator, a pulse forming network, two broadband horn antennas for transmission, and reception, as well as a sampling oscilloscope for data acquisition. A photo of the test setup is shown in Figure 5.

For UWB radar systems, it is very important to obtain a large bandwidth to achieve a high-resolution capability. One way to obtain a large bandwidth, and at the same time keeping the centre frequency low, is to let the lower band edge approach zero. This type of pulse is called a baseband pulse, and systems employing such pulses are called impulse radars. To occupy a large bandwidth, the pulse must be extremely short, typically nanoseconds or below. Therefore, in pulsebased UWB systems, Gaussian monocycle pulse generators 
are mainly used at the transmitter stage [11]. These transmitted pulses will be affected by the illuminated material, the reflection behavior of the target, as well as the used antennas, producing a derivative of the driving Gaussian monocycle pulse resulting in a shape of Ricker wavelet.

To show the performance of the proposed CVPP model, a measured UWB pulse, which was reflected on the chest of a human body, was used, shown in Figure 6. Furthermore, this figure depicts the signal as reconstructed using the Ricker wavelet, the Hilbert transform of the Ricker wavelet, as well as a CVPP formed by the Ricker wavelet. The modulation angle for the CVPP is calculated to $\varphi_{c}=53.2^{\circ}$. It appears that the best match with the measured signal is achieved with the real part of the CVPP; whereas the Ricker wavelet or the Hilbert transform of the Ricker wavelet shows significant deviations in parts of the signal.

In addition, the Hilbert transform of the measured signal and the imaginary part $\operatorname{Im}\left\{f_{c}(t)\right\}$ of the CVPP which was used to reconstruct the measured pulse can be seen in Figure 7. Furthermore, this figure includes the signal reconstruction of the Hilbert transform of the measured UWB radar signal using a Ricker wavelet as well as the Hilbert transform of the Ricker wavelet. Again, the CVPP is a very good approximation for the basic shape of the pulse; whereas the real-valued wavelets show large differences between the signal and the reconstruction in particular in the pre- and postoscillations.

This example illustrates that for pulse reconstruction from measured signals, the use of hybrid pulse forms is essential, since pre- and postoscillations in the reconstruction contain important information about object characteristics such as shape and dimension [12].

A contour plot of the absolute value of the WT of the measured signal using a Ricker wavelet for analysis is shown in Figure 8. The WT shows several major side maxima, even if the pulse shape is identical with the basic shape of the mother wavelet. The characteristic using the Hilbert transform of the Ricker wavelet for signal analysis would be very similar.

A contour plot of the absolute value of the WT of the analytic measured signal using an analytic Ricker wavelet for analysis is shown in Figure 9. As we can see, the WT shows almost no undesired side maxima. Due to a bad localization of the Ricker wavelet in frequency domain, the maxima of the WT extends over a wide range of scales.

\section{CONCLUSION}

For pulse analysis, classic correlation-based methods like the real-valued matched filter concept require the exact knowledge of the basic pulse shape to determine position or intensity. The real-valued WT can handle shifted and stretched versions of a mother wavelet, but still the basic shape of the pulse is required.

The concept of CVPP gives us the ability to directly model the basic shape of a pulse by using the modulation angle parameter. Furthermore, this concept enables a highly advanced pulse reconstruction especially for applications

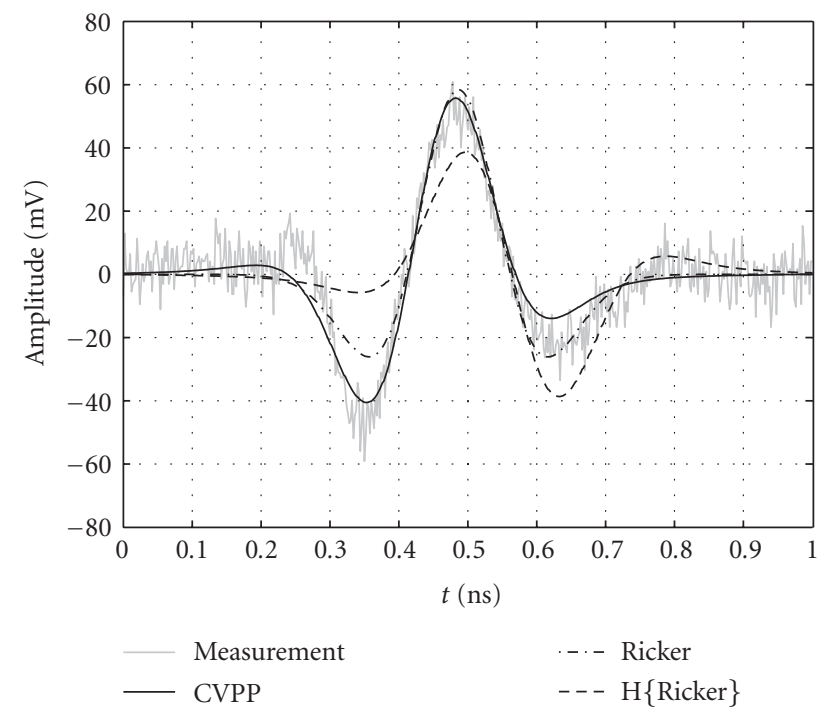

FIGURE 6: Measurement of a UWB pulse which was reflected on the chest of a human as well as the real-valued reconstructed pulse by using a CVPP, the Ricker wavelet, and the Hilbert transform of the Ricker wavelet.

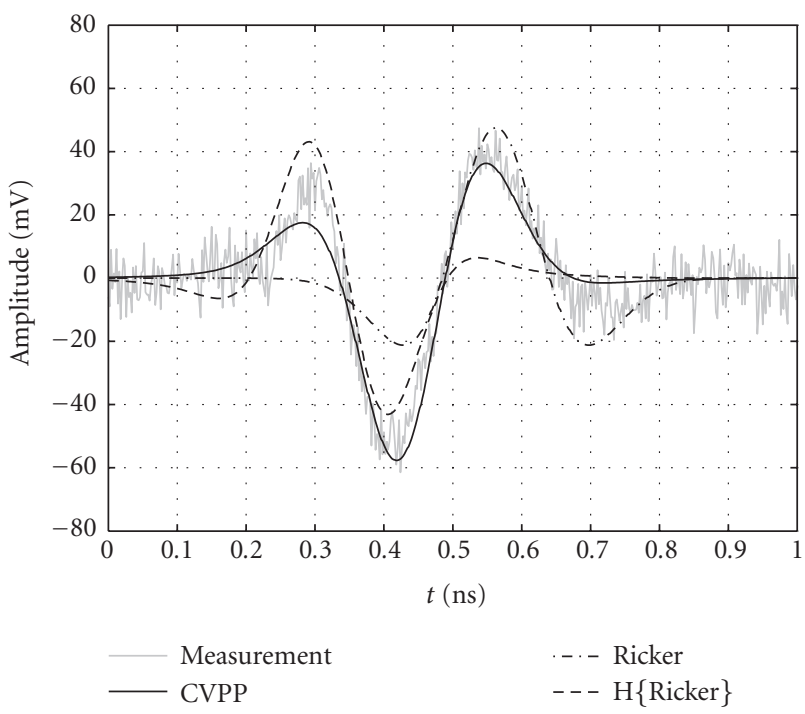

FIGURE 7: Hilbert transform of measured UWB radar pulse as well as the real-valued reconstructed pulse by using a CVPP, the Ricker wavelet, and the Hilbert transform of the Ricker wavelet.

with little or no knowledge of the transmission behavior of the propagation medium and the reflection behavior of the target, because the influence of the basic shape of the basic wavelet is far less important compared to the real-valued case. The results of the complex-valued pulse parameter estimation can be used in constructing a matched filter for correlation-based receiver design. The proof of this concept is demonstrated on synthetic data and also carried out on UWB pulse measurement data. 


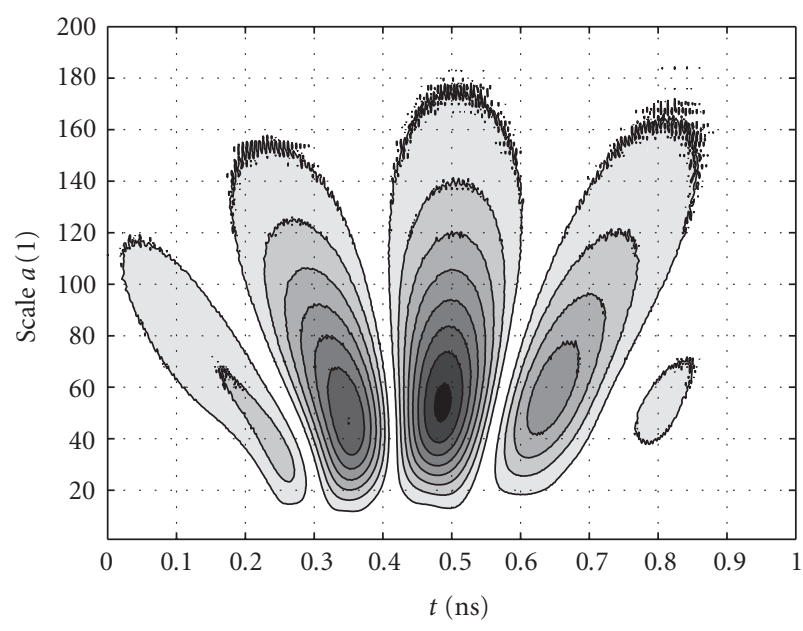

FIGURE 8: Contour plot of the absolute value of the WT of the measured signal using a Ricker wavelet for analysis.

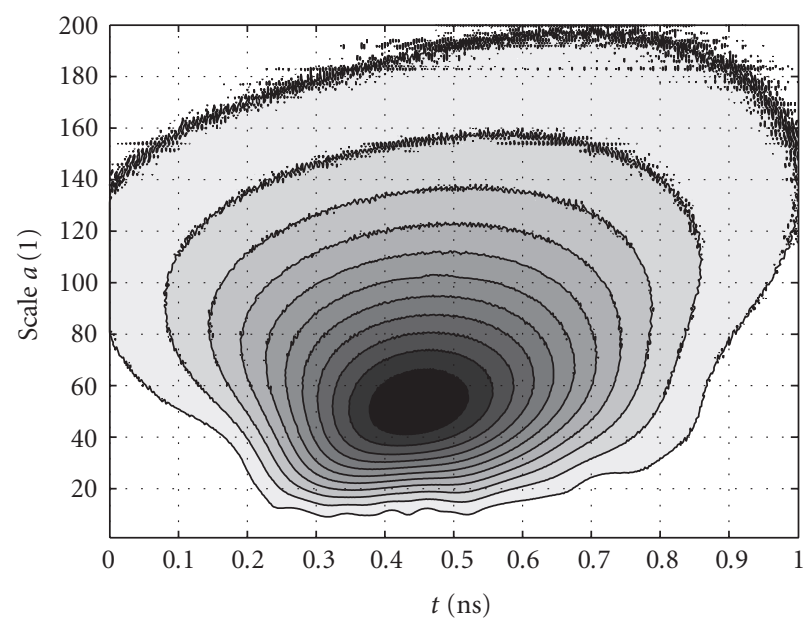

FIgURE 9: Contour plot of the absolute value of the WT of the analytic measured signal using an analytic Ricker wavelet for analysis.

\section{ACKNOWLEDGMENTS}

The authors would like to thank M. Pichler, E. Schimbäck, and T. Buchegger of the Linz Center of Competence in Mechatronics for fruitful discussions and the assistance in gaining measurement data.

\section{REFERENCES}

[1] S. L. Hahn, Hilbert Transforms in Signal Processing, Artech House, Norwood, Mass, USA, 1996.

[2] A. Grossman and J. Morlet, "Decomposition of Hardy functions into square integrable wavelets of constant shape," SIAM Journal on Mathematical Analysis, vol. 15, no. 4, pp. 723-736, 1984.

[3] I. Daubechies, Ten Lectures on Wavelets, SIAM, Philadelphia, $\mathrm{Pa}, \mathrm{USA}, 1992$.

[4] S. Mallat, A Wavelet Tour of Signal Processing, Academic Press, New York, NY, USA, 2nd edition, 1998.
[5] A. D. Poularikas, The Transforms and Applications Handbook, CRC Press LLC, Boca Raton, Fla, USA, 2000.

[6] D. E. Iverson, "Coherent processing of ultra-wideband radar signals," IEE Proceedings - Radar, Sonar and Navigation, vol. 141, no. 3, pp. 171-179, 1994.

[7] L. Y. Astanin and A. A. Kostylev, Ultrawideband Radar Measurements Analysis and Processing, IEEE Press, London, UK, 1997.

[8] C. Chambers, S. R. Cloude, P. D. Smith, A. Milne, K. Trafford, and D. M. Parkes, "Wavelet processing of ultra wideband radar signals," in Proceedings of IEE Colloquium on Antenna and Propagation Problems of Ultrawideband Radar, pp. 1/11/4, London, UK, January 1993.

[9] K. Pourvoyeur, A. Stelzer, G. Ossberger, T. Buchegger, and M. Pichler, "Wavelet-based impulse reconstruction in UWBradar," in Proceedings of IEEE MTT-S International Microwave Symposium Digest (IMS '03), vol. 1, pp. 603-606, Philadelphia, $\mathrm{Pa}$, USA, June 2003.

[10] B. Scheers, Y. Plasman, M. Acheroy, and A. Vander Vorst, "Laboratory UWB GPR system for landmine detection," in Proceedings of the 8th International Conference on GroundPenetrating Radar (GPR '00), pp. 747-752, Gold Coast, Australia, May 2000.

[11] T. Buchegger, G. Oßberger, C. Diskus, A. Springer, and A. Stelzer, "A novel low-cost ultra wideband microstrip pulse forming network for Gaussian monocycle generation," in Proceedings of the International Workshop on Ultra Wideband Systems (IWUWBS '03), Oulu, Finland, June 2003.

[12] G. Oßberger, T. Buchegger, K. Pourvoyeur, E. Schimback, A. Stelzer, and R. Weigel, "Ultra-wideband object characterization via A-scan," in Proceedings of IEEE Conference on Ultra Wideband Systems and Technologies (UWBST '03), pp. 478482, November 2003.

Klaus Pourvoyeur was born in Ehenbichl, Austria, in 1977. He received the Dipl. Ing. (M.S.) degree in mechatronics from Johannes Kepler University, Linz, Austria, in 2005. He was with the Institute for Communications and Information Engineering at the University of Linz, and joined the Linz Center of Competence in Mechatronics, Linz, Austria, in 2004 for his Ph.D. thesis. His research interests are pulse analysis for UWB radar as well as position measurement for real-time applications.

Andreas Stelzer was born in Haslach an der Mühl, Austria, in 1968. He received the Engineer Diploma degree in electrical engineering from the Technical University of Vienna, Austria, in 1994. In 2000, he received the Dr. Techn. degree (Ph.D.) in mechatronics with honors "sub auspiciis praesidentis rei publicae" from the Johannes Kepler University, Linz. In 2003, he finished his habilitation thesis and became an As-

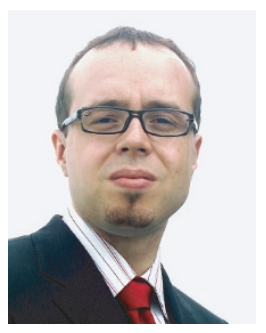
sociate Professor at the Johannes Kepler University, Linz. He joined the Johannes Kepler University as a University Assistant in 1994. Since 2000, he has been with the Institute for Communications and Information Engineering. His research work focuses on microwave sensor systems for industrial applications, RF and microwave subsystems, SAW sensor systems and applications, 
as well as digital signal processing for sensor signals. He has authored or coauthored more than 100 journals and conference papers. He is a Member of the Austrian OVE, and an IEEE Member of Microwave Theory and Techniques, Circuits and Systems, and Instrumentation and Measurement Society. He served as an Associate Editor for the IEEE Microwave and Wireless Components Letters.

Gerald Oßberger was born in Linz, Austria, in 1975. He received the Dipl. Ing. (M.S.) degree in mechatronics engineering from Johannes Kepler University, Linz, Austria, in 2002. In 2002, he joined the Linz Center of Mechatronics GmbH for his Ph.D. thesis. His thesis treats design, simulation, measurement, and signal processing of an ultrawideband radar application.

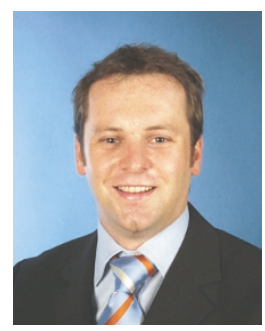

